\title{
CORRESPONDENCE
}

\section{Limitations of calculating "true" regression slope: impact on estimates of minimal important difference}

\section{To the Editors:}

We read with interest the recent article [1] that addresses the important question of minimal important difference (MID) for exercise tests in chronic obstructive pulmonary disease. PUHAN et al. [1] use both distribution- and anchor-based methods, and it is the anchor-based methodology that we would like to consider here. Those authors use linear regression analysis between change in the dependent variable, in this case 6-min walk distance, and change in an anchor variable (St George's Respiratory Questionnaire and University of California San Diego Shortness of Breath Questionnaire) as the independent variable. We would like to highlight limitations of this approach and demonstrate, with example data, the potential impact on estimates of MID. The problem is based on the assumption that the independent variable $(X)$ is measured without error, but the slope of linear regression will be a measure of the "true" relationship only if all variance of $X$ is due to the regression and not due to measurement error. Although no measurement is free from error, some signals are rather more "noisy" than others. The presence of high correlation between the variables will indicate the reduction in such a potential error. This point is made by the authors, who suggest an r-value of 0.5 as a cut-off and a linear relationship between the two variables.

Figures 1 and 2, and tables 1 and 2 show a worked example using fictional data of two linear and strongly correlated $(\mathrm{r}=0.76)$ variables, $A$ and $B$. The MID for $B$ is known to be 2; this example will illustrate the impact of switching variables on the $x$ - and $y$-axes of the scatterplot on estimating the MID of variable $A$.

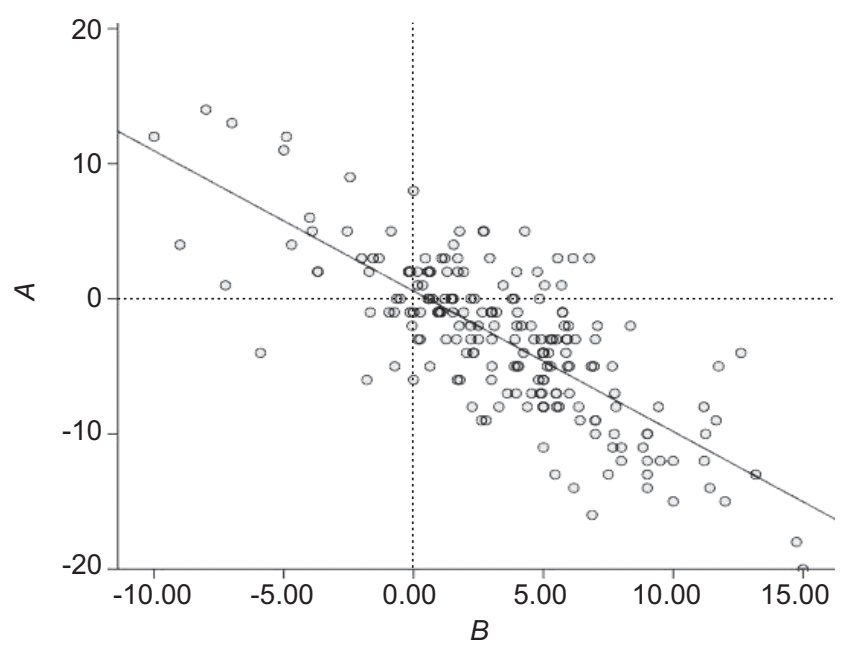

FIGURE 1. Scatter plot of variable $A$ versus variable $B$. Linear $r^{2}=0.582$
This example demonstrates that estimates of MID vary considerably $(-0.4$ versus -1.5$)$ despite a high correlation coefficient $\mathrm{r}=0.76$, well above the threshold suggested by PUHAN et al. [1].

The problem of measurement error when comparing two variables has been discussed at length in work by DAUBENSPECK and OGDen [2], Kendall [3], and KeRMACK and Haldane [4]. They propose that this may be resolved by either normalising one axis or dividing each observation by its standard deviation, estimated from the pooled observations. This approach would eliminate the dependency of the fitted line parameters on the scale of the measurement used; however, it would not readily allow estimation of an MID. The message is that mapping exercises such as this become less reliable with lower correlations between the two variables that are being compared and, even when those variables are well correlated, may lead to an unreliable estimate of the MID. MID will be estimated most reliably though direct measurement rather than statistical inference.

\section{J.W. Dodd and P. Jones}

Dept of Respiratory Medicine, Clinical Sciences, St George's University of London, London, UK.

Correspondence: J.W. Dodd, Dept of Respiratory Medicine, Clinical Sciences, St George's University of London, Cranmer Terrace, London SW17 0RE, UK. E-mail: jdodd@sgul.ac.uk

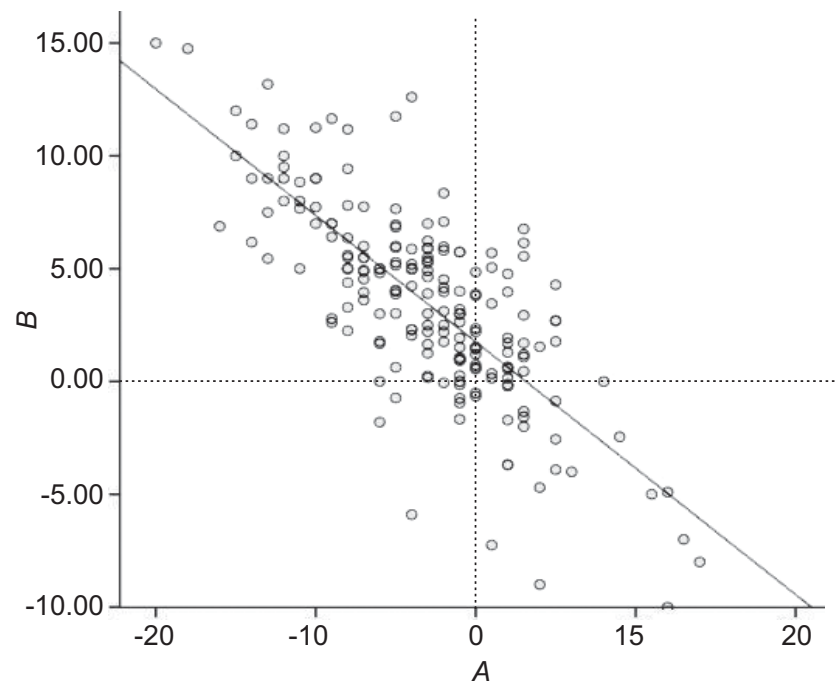

FIGURE 2. Scatter plot of variable $B$ versus variable $A$. Linear $r^{2}=0.582$. 


\begin{tabular}{|c|c|c|c|c|c|}
\hline TABLE 1 & $\begin{array}{l}\text { Linear regr } \\
\text { dependent }\end{array}$ & $\begin{array}{l}\text { on an } \\
\text { iable }\end{array}$ & ysis with vari & able $A$ a & s the \\
\hline Model & \multicolumn{3}{|c|}{ Coefficients } & \multirow[t]{3}{*}{$\mathbf{t}$} & \multirow[t]{3}{*}{ Sig. } \\
\hline & \multicolumn{2}{|c|}{ Unstandardised } & \multirow{2}{*}{$\frac{\text { Standardised }}{\beta}$} & & \\
\hline & B & SE & & & \\
\hline (Constant) & 0.568 & 0.342 & & 1.663 & 0.098 \\
\hline Variable $B$ & -1.039 & 0.063 & -0.763 & -16.606 & 0.000 \\
\hline
\end{tabular}

\begin{tabular}{|c|c|c|c|c|c|c|}
\hline \multicolumn{2}{|c|}{ TABLE 2} & \multicolumn{5}{|c|}{$\begin{array}{l}\text { Linear regression analysis with variable } B \text { as the } \\
\text { dependent variable }\end{array}$} \\
\hline \multicolumn{2}{|c|}{ Model } & \multicolumn{3}{|c|}{ Coefficients } & \multirow[t]{3}{*}{$\mathrm{t}$} & \multirow[t]{3}{*}{ Sig. } \\
\hline & & \multicolumn{2}{|c|}{ Unstandardised } & \multirow{2}{*}{$\frac{\text { Standardised }}{\beta}$} & & \\
\hline & & B & SE & & & \\
\hline \multirow[t]{2}{*}{1} & (Constant) & 1.758 & 0.220 & & 8.005 & 0.000 \\
\hline & Variable $A$ & -0.560 & 0.034 & -0.763 & -16.606 & 0.000 \\
\hline
\end{tabular}

Statement of Interest: Statements of interest for both authors can be found at www.erj.ersjournals.com/site/misc/statements. xhtml

\section{REFERENCES}

1 Puhan MA, Chandra D, Mosenifar Z, et al. The minimal important difference of exercise tests in severe COPD. Eur Respir J 2011; 37: 784-790.

2 Daubenspeck JA, Ogden RD. Estimation of response slopes in respiratory control using directional statistics. J Appl Physiol 1978; 45: 823-829.

3 Kendall MG. A Course in Multivariate Analysis. London, Giffin 1957.

4 Kermack KA, Haldane JBS. Organic correlation and allometry. Biometrika 1950; 37: 30-41.

DOI: $10.1183 / 09031936.00170910$

\section{From the authors:}

J.W. Dodd and P. Jones argue that anchor-based approaches may lead to unreliable estimates of the minimal important difference (MID) because of measurement error. Using simulated data, they show that MID estimates may vary considerably even if there is a strong correlation between the anchor measures and the outcome of interest (6-min walk distance in our study). Thereby, they illustrate the properties of the correlation coefficient, which does not change if one swaps the $x$ and $y$ variable, and of the fitting line, which changes depending on whether $x$ predicts $y$ or $y$ predicts $x$.

We agree that anchor-based approaches have their limitations and, therefore, used several anchors in our analyses, as well as distribution-based approaches. We are, however, not entirely clear as to how J.W. Dodd and P. Jones define measurement error and what they mean by direct methods. We believe that they refer to a question of validity of the anchor rather than measurement error. In statistics, measurement error refers to a single variable whose measurement is prone to some random variability (random error) and perhaps some systematic error. A correlation coefficient, instead, represents a measure for how closely a variable such as an anchor relates to the outcome of interest. Both measurement error and validity of the anchor influence MID estimates. Measurement error can be taken into consideration in the analyses and usually biases estimates towards an underestimation. If we assume, for example, an intraclass correlation coefficient of 0.95 for repeated measurements of the total score of the St George's Respiratory Questionnaire, as reported previously [1], the MID adjusted for the small measurement error (for example using the "eivreg" command of STATA) would be 25.4 , instead of the 24.6 as reported in our paper [2]. If the intraclass correlation coefficient was only 0.8 , the MID estimate would be 29.3, indicating that an unadjusted MID estimate indeed represents an underestimation of the MID if the anchor is not measured with high reliability.

Options are limited if the anchor is not a valid measure for the outcome of interest. Some authors have proposed correlations coefficients of $\geqslant 0.3$ to be sufficient to derive MID estimates [3], but we believe that such a cut-off is too lenient. In our analysis, the strong correlation between the anchor and the outcome of interest of $\geqslant 0.5$, as well as the use of multiple anchors, increases our confidence in the reported estimate. In addition, as explained previously, the measurement error of the anchor can be taken into consideration if the intraclass correlation coefficient for measuring the anchor is $<0.9$ in order to avoid underestimation of the MID. We believe that these two approaches, used in our analysis, protect against unreliable MID estimates.

\section{M.A. Puhan*, D. Chandra", R.A. Wise ${ }^{*}$ and F. Sciurba"}

*Dept of Epidemiology, Johns Hopkins Bloomberg School of Public Health, "Dept of Medicine, Johns Hopkins University, Baltimore, MD, and "Dept of Pulmonary, Allergy and Critical Care Medicine, University of Pittsburgh, Pittsburgh, PA, USA.

Correspondence: M.A. Puhan, Dept of Epidemiology, Johns Hopkins Bloomberg School of Public Health, 615 North Wolfe Street, Baltimore, MD, USA. E-mail: mpuhan@jhsph.edu

Statement of Interest: None declared.

\section{REFERENCES}

1 Wilson CB, Jones PW, O'Leary CJ, et al. Validation of the St. George's Respiratory Questionnaire in bronchiectasis. Am J Respir Crit Care Med 1997; 156: 536-541.

2 Puhan MA, Chandra D, Mosenifar Z, et al. The minimal important difference of exercise tests in severe COPD. Eur Respir J 2011; 37: 784-790.

3 Revicko D, Hays RD, Cella D, et al. Recommended methods for determining responsiveness and minimally important differences for patient-reported outcomes. J Clin Epidemiol 2008; 61: 102-109.

DOI: 10.1183/09031936.00179610 